\title{
Does Inflation Provide Natural Initial Conditions for the Universe? ${ }^{1}$
}

\author{
Sean M. Carroll and Jennifer Chen \\ Enrico Fermi Institute, Department of Physics, and \\ Kavli Institute for Cosmological Physics, University of Chicago \\ 5640 S. Ellis Avenue, Chicago, IL 6063\%, USA \\ carroll@theory.uchicago.edu, jennie@theory.uchicago.edu
}

\begin{abstract}
If our universe underwent inflation, its entropy during the inflationary phase was substantially lower than it is today. Because a low-entropy state is less likely to be chosen randomly than a high-entropy one, inflation is unlikely to arise through randomlychosen initial conditions. To resolve this puzzle, we examine the notion of a natural state for the universe, and argue that it is a nearly-empty spacetime. If empty space has a small vacuum energy, however, inflation can begin spontaneously in this background. This scenario explains why a universe like ours is likely to have begun via a period of inflation, and also provides an origin for the cosmological arrow of time.
\end{abstract}

\footnotetext{
${ }^{1}$ Submitted for consideration in the Gravity Research Foundation Essay Competition. Based on hep-th/0410270 This work was supported in part by the U.S. Dept. of Energy, the National Science Foundation, the NDSEG Fellowship, and the David and Lucile Packard Foundation.
} 
As far as we know, there is only one universe. At least, there are not multiple universes between which we can easily travel; we only have access to the universe we are in, and (because there is a finite time back to a dense early stage past which we can't see) we can only experience a finite portion of that.

So we are stuck with the universe, and in principle we simply have to accept the conditions we see. Nevertheless, there is an irresistible temptation to try to explain the state in which we find our universe as the practically-inevitable outcome of some dynamical processes. The most celebrated attempt along these lines has been the theory of inflation [1, 2, 3], which purports to explain the flatness, homogeneity, and isotropy of our universe through an early period of accelerated expansion.

In explaining how inflation accounts for these features of our observed universe, cosmologists often appeal to a certain intuitive notion of what would constitute a "natural" or "randomly-chosen" initial condition, understanding that these concepts are not precisely defined. It is imagined that natural conditions for the early universe would involve large fluctuations of energy density and spacetime curvature, randomly distributed through space 4]. Occasionally, a patch of space will appear in which the conditions are right for inflation to begin: a smooth configuration dominated by vacuum energy over a region larger than the local Hubble radius [5]. Once inflation begins, that small patch can grow to the size of our observable universe.

However, this notion of "natural" cannot be right. One way to see this is to consider the entropy of the universe at different stages of its evolution. Within our current Hubble patch, the entropy due to particles is of order $S_{M}(U) \sim 10^{88}$. This was the dominant contribution to the entropy of our comoving Hubble patch at early times. Today, however, the entropy is dominated by black holes [6]. The Bekenstein-Hawking entropy of a black hole is proportional to its horizon area, $S_{B H}=A / 4 G \sim 10^{77}\left(M_{B H} / M_{\odot}\right)^{2}$. Since there are probably more than ten billion galaxies in the observable universe with million-solar-mass black holes at their centers, the current entropy in black holes is at least $S_{B H}(U) \sim 10^{99}$.

Now let us compare this number to the entropy at the beginning of inflation. If inflation happens at an energy scale $M_{I} \sim 10^{15} \mathrm{GeV}$, the Hubble radius that grew into our observable universe was $H_{I}^{-1} \sim 10^{6} L_{P}$, where $L_{P} \sim 10^{18} \mathrm{GeV}^{-1}$ is the Planck length. The amount of entropy in this proto-inflationary patch is approximately the entropy of an horizon volume 
of a de Sitter spacetime with the same Hubble parameter, $S_{I} \sim\left(H_{I}^{-1} / L_{P}\right)^{2} \sim 10^{12}$.

The entropy of the proto-inflationary patch, then, is fantastically smaller than the entropy of our current Hubble volume, or even than that of our comoving volume at early times before there were any black holes. This is in perfect accord with the Second Law of Thermodynamics, since the entropy is increasing. But it is hard to reconcile with the idea that we should find an appropriate proto-inflationary patch within the randomly fluctuating early universe. If we are randomly choosing conditions, it is much easier to choose high-entropy conditions than low-entropy ones; hence, it would much more likely to simply find a patch that looks like our universe today, than to find one that was about to begin inflating.

This point is somewhat counterintuitive, and worth emphasizing. Despite their vast differences in size, energy, and number of particles, the proto-inflationary patch and our current universe are two configurations of the same system, since one can evolve into the other. There are many more ways for that system to look like our current universe than to be in a proto-inflationary configuration. (See also [6, 17, 8, 9, 10, 11, 12.)

Therefore, if inflation is to help explain the conditions of our universe, there must be some reason why it started. To understand what this reason might be, we should re-examine the notion of naturalness. Without a microscopic understanding of the statistical mechanics of quantum gravity, we can nevertheless appeal to the fact that systems generically evolve to high-entropy states. In other words, we should ask what kind of ultimate state we expect the universe to evolve towards.

In the absence of gravity, high-entropy states tend to become homogeneous. But in the presence of gravity, the Jeans instability can lead to increased inhomogeneity, and eventually to black holes [6]. But a collection of black holes is not stable; spacetime will typically be either expanding or contracting (locally), and each black hole will eventually evaporate via Hawking radiation. Therefore, unless the state is arranged so that the entire universe crunches into a future singularity, spacetime will tend towards a state which is nearly empty. General relativity allows us to increase the entropy of nearly any state by increasing the volume of space and scattering the constituents to the far corners of the universe.

But even empty space can have energy, and recent cosmological observations have presented strong evidence for a small nonzero vacuum energy [13, 14]. In the presence of such an energy, empty space will settle into a de Sitter configuration; the natural state for the 
universe to be in is therefore a nearly-empty de Sitter space [15].

Unlike Minkowski space, de Sitter has a nonzero temperature; for realistic parameters, this temperature is $T_{\mathrm{dS}} \sim H_{0} \sim 10^{-33} \mathrm{eV}$. In the presence of an appropriate inflaton field, thermal fluctuations will occasionally conspire to produce a tiny, smooth region of space dominated by a large vacuum energy - the correct conditions for a proto-inflationary patch 15. This patch can then inflate and reheat to produce a universe like ours. An observer in the background would simply see the formation of a small black hole that would eventually radiate away. (See also [16, 17, 18, 19, 20, 21].)

Of course, thermal fluctuations in a de Sitter background can produce all sorts of things, including a non-inflating Robertson-Walker universe. However, the fluctuations are not "randomly chosen" in the measure defined by the entropy; they arise from a very specific condition, provided by the background de Sitter space. Because the entropy density of the background is so low, it is easier to fluctuate into a small proto-inflationary patch than into a universe that looks like ours today.

This story, in which inflation arises via a thermal fluctuation in a background de Sitter spacetime, bears a resemblance to the idea that the universe arises as a quantum fluctuation "from nothing" [22, 23, 24, 25, 26]. There is an important advantage to the current scenario, however: it provides a natural explanation for the cosmological arrow of time [27, 28]. The arrow-of-time puzzle can be simply stated: why are the initial conditions of the universe so different from the final conditions? In the creation-from-nothing picture, this problem is especially acute; boundary conditions are imposed at early times but not at late times.

For our picture [15], in contrast, early and late times have the same structure in the wider universe: a background de Sitter geometry that occasionally nucleates a small inflating patch (cf. [29, 30]). Starting from a Cauchy surface with generic initial data, evolution to both the past and future will feature emptying-out to a de Sitter state, followed by eventual fluctuations into new inflating universes. The total entropy of the universe will increase because it always can increase; there is no equilibrium configuration in which entropy is maximized. The mechanism for entropy increase is the creation of new inflating patches, which can eventually evolve into universes like ours; the universe appears time-asymmetric to us because we can only see a tiny piece of it.

We therefore believe that inflation does provide natural initial conditions for the universe 
we see, once we place it in the proper context of a larger spacetime that is stubbornly trying to increase its entropy. It is hard to think of any directly observable consequences of this scenario, but understanding the conceptual underpinnings of inflation is an important part of making sense of the universe we do observe.

\section{References}

[1] A. H. Guth, Phys. Rev. D 23, 347 (1981).

[2] A. D. Linde, Phys. Lett. B 108, 389 (1982).

[3] A. Albrecht and P. J. Steinhardt, Phys. Rev. Lett. 48, 1220 (1982).

[4] A. D. Linde, Phys. Lett. B 129, 177 (1983).

[5] T. Vachaspati and M. Trodden, Phys. Rev. D 61, 023502 (2000) arXiv:gr-qc/9811037.

[6] R. Penrose, The Emperor's New Mind, ch. 7 (Oxford: Oxford University Press, 1989), and references therein.

[7] P. C. W. Davies, Nature 301, 398 (1983).

[8] D. N. Page, Nature 304, 39 (1983).

[9] W. G. Unruh, Published in *Princeton 1996, Critical dialogues in cosmology* 249-264.

[10] S. Hollands and R. M. Wald, Gen. Rel. Grav. 34, 2043 (2002) arXiv:gr-qc/0205058.

[11] L. Dyson, M. Kleban and L. Susskind, JHEP 0210, 011 (2002) arXiv:hep-th/0208013.

[12] A. Albrecht and L. Sorbo, arXiv:hep-th/0405270.

[13] A. G. Riess et al. [Supernova Search Team Collaboration], Astron. J. 116, 1009 (1998) arXiv:astro-ph/9805201.

[14] S. Perlmutter et al. [Supernova Cosmology Project Collaboration], Astrophys. J. 517, 565 (1999) arXiv:astro-ph/9812133.

[15] S. M. Carroll and J. Chen, arXiv:hep-th/0410270.

[16] E. Farhi and A. H. Guth, "An Obstacle To Creating A Universe In The Laboratory," Phys. Lett. B 183, 149 (1987).

[17] A. Vilenkin, Phys. Rev. D 37, 888 (1988).

[18] E. Farhi, A. H. Guth and J. Guven, Nucl. Phys. B 339, 417 (1990).

[19] W. Fischler, D. Morgan and J. Polchinski, Phys. Rev. D 41, 2638 (1990).

[20] W. Fischler, D. Morgan and J. Polchinski, Phys. Rev. D 42, 4042 (1990). 
[21] A. D. Linde, Nucl. Phys. B 372, 421 (1992) arXiv:hep-th/9110037.

[22] A. Vilenkin, Phys. Lett. B 117, 25 (1982).

[23] A. Vilenkin, Phys. Rev. D 27, 2848 (1983).

[24] J. B. Hartle and S. W. Hawking, Phys. Rev. D 28, 2960 (1983).

[25] A. D. Linde, Lett. Nuovo Cim. 39, 401 (1984).

[26] A. Vilenkin, Phys. Rev. D 30, 509 (1984).

[27] H. Price, in Time's Arrows Today, ed. S. Savitt (Cambridge: Cambridge University Press, 1994); arXiv:gr-qc/9310022.

[28] A. Albrecht, arXiv:astro-ph/0210527.

[29] J. Garriga and A. Vilenkin, Phys. Rev. D 57, 2230 (1998) arXiv:astro-ph/9707292.

[30] A. Aguirre and S. Gratton, Phys. Rev. D 67, 083515 (2003) arXiv:gr-qc/0301042. 